\title{
PERSPECTIVAS DEL CAD EN ARQUITECTURA E INGENIERIA
}

\author{
(PERSPECTIVES OF CAD IN ARCHITECTURE AND ENGINEERING)
}

\author{
A. Recuero, Dr. Ingeniero de Caminos \\ IETCC/CSIC
}

\begin{abstract}
RESUMEN
En este articulo se analizan, brevemente, la situación actual y las perspectivas, para un próximo futuro, de la aplicación de los sistemas de CAD en el campo de la construcción. Se considera que el CAD debe abordar, de modo integrado, el proceso completo de proyecto, incluyendo no sólo los aspectos de síntesis, sino también los de análisis y evaluación.

Se señalan los puntos en los que es preciso conicentrar los esfuerzos de investigación y desarrollo. Estos puntos son:

- Comunicación usuario-computador.

- Estructura de las bases de datos.

- Coordinación de las distintas profesiones que intervienen en el hecho constructivo, para definir las necesidades a cubrir y el grado de integración deseable.
\end{abstract}

Se señala, asimismo, la necesidad de establecer métodos y criterios para verificar la corrección de las soluciones propuestas por estos programas.

\section{SUMMARY}

In this paper, present and short term situations of CAD for tuildings are analyzed. It is assumed that CAD must cover the design process in its entirety not only the synthesis aspects, but also those of analysis and evaluation.

Research and Development efforts should be undertaken in the following:

- Computer-user interfaces.

- Data base structures.

- Coordination of the various related professions, in order to define their needs, and the degree of integration to be achieved.

- Establishment of methods and criteria to check for the adequacy of the solutions provided by these systems.

\section{Introducción}

El significado de las siglas CAD (Computer Aided Design) ha ido evolucionando a lo largo de los años. Inicialmente se consideraba, prácticamente, sinónimo de Análisis de Estructura por el método de Elementos Finitos. Posteriormente se asoció al dibujo asistido por computador y, en la actualidad, se asocia, generalmente, con el diseño de objetos tridimensionales. Actualmente se utiliza, también, las siglas CAE (Computer Aided Engineering) como una disciplina más general que incluye todos los tipos de ayudas que puede prestar el computador en el desarrollo de proyectos.

En lo que sigue se utilizará el término CAD con un significado amplio que cubre los aspectos relacionados con equipos, programas, análisis de sistemas y la me- todología de Ingenieria y Arquitectura destinadas a realizar el proyecto completo de construcciones, utilizando los computadores.

Con frecuencia aparecen asociadas las siglas CAD y CAM (Computer Aided Manufacturing); sin embargo, deben considerarse como disciplinas independientes, aunque relacionadas. De hecho, los datos de partida para CAM, que deben ser interpretados por máquinasherramienta, pueden ser producidos mediante un sistema CAD durante el proceso de diseño, pero el CAM no forma parte del proceso de diseño.

El proyecto no es meramente la creación de nuevos objetos, más o menos conducida por la creatividad del proyectista, sino que incluye, también, el análisis del comportamiento mecánico, las características físicas, 
la adecuación al plan de necesidades, la presentación de resultados para el posterior control o ejecución, la simulación de diversas situaciones e, incluso, la optimización. Todas estas herramientas deben estar al servicio de un proceso iterativo, cuyo objetivo es conseguir proyectos factibles y optimizados, en la medida de lo posible.

\section{Historia del CAD}

Una prueba histórica de que el CAM no es un descendiente del CAD, sino que tiene orígenes propios diferenciados, es el que ya a principios de los años 50 se desarrolló en el Instituto Tecnológico de Massachusetts (MIT) la primera máquina-herramienta controlada automáticamente por un computador.

Sutherland, también del MIT, considerado como uno de los pioneros del CAD, desarrolló en 1963 el sistema SKETCHPAD en el que ya introdujo ideas utilizadas actualmente, como el cursor elástico, los círculos de influencia y operaciones de ampliación, rotación de objetos, sistemas locales de referencia, etc. En 1964 se introdujo en General Motors el sistema de DAC1, soportado sobre máquinas IBM, dedicado al dibujo de planos, si bien no incorporaba técnicas gráficas interactivas.

En 1965 los Laboratorios Bell Telephone anunciaron la Pantalla Graphic 1, modificación de una pantalla DEC y con un controlador PDP-5. Este sistema se utilizó para el diseño de circuitos impresos, creación y edición de textos, elaboración de diagramas de bloques, diseño de cableados, etc. Esta pantalla puede considerarse como uno de los primeros pasos en el proceso, hoy generalizado, de tener las potencialidades del CAD, distribuidas en estaciones de trabajo interactivas y, especialmente dedicadas, conectadas con un sistema central.

En 1967 Freeman sugirió uno de los primeros algoritmos para la consideración de partes vistas y ocultas en el dibujo de los objetos. La primera mitad de los años 70 fue una época de gran entusiasmo entre los científicos y los informáticos respecto a la implementación de sistemas de CAD. En este período se realizó un importante trabajo teórico y se pusieron las bases del CAD, tal como hoy lo entendemos. Así, se desarrolló el sistema ICES (Integrated Civil Engineering System), en el que incorporaron una gran parte de las ideas del CAD. También, por estos años se produjo el boom de los programas de análisis de estructuras por el método de Elementos Finitos y se progresó en el tema de representación gráfica de superficies y en el de la eliminación de partes ocultas.

De entonces hacia aqui se han producido notables desarrollos, sobre todo en lo referente a sistemas de di- bujo, lo que ha contribuido a asignar al término $C A D$ el sentido restringido citado al principio. Como ya se ha dicho anteriormente, preferimos considerar incluidos dentro del CAD todos los aspectos de síntesis, análisis y evaluación de los proyectos.

Los arquitectos e ingenieros se ven cada vez más abocados a utilizar en su trabajo las herramientas informáticas, debido, por un lado, a la presión de factores económicos y, por otro, a la posibilidad de liberarse de las labores más rutinarias y repetitivas; sin embargo, las tendencias en el ambiente técnico no están siempre dirigidas en la dirección adecuada y, de hecho, la conjunción de los intereses comerciales de las firmas que desarrollan los programas y equipos, y los factores económicos generales han conducido a una situación confusa que, incluso, puede calificarse de caótica en algunos aspectos. Vamos a tratar de analizar, de forma breve, los aspectos más relevantes de la actual situación.

\section{Herramientas informáticas de carácter general}

Los medios informáticos utilizados en las oficinas de proyecto pueden agruparse en herramientas de carácter general y en herramientas de diseño asistido. Dentro de las primeras, pueden considerarse los procesadores de textos, los programas de creación y mantenimiento de bases de datos, las hojas electrónicas, programas estadísticos, todo tipo de programas matemáticos, programas de gestión contable, de preparación y control de presupuestos, etc.

Es posible encontrar este tipo de programas prácticamente para cualquier equipo disponible. Con gran frecuencia se adquiren como un primer paso, dentro de un plan de automatización de la oficina; pero, en muchas ocasiones, estos planes están, en realidad, poco o nada desarrollados. Es habitual alcanzar muy pronto las limitaciones de los programas o de los equipos que los soportan, lo que conduce a un primer descon. cierto. Además, en la mayor parte de los casos, se juzga que la consulta a especialistas es excesivamente cara, cuando, en realidad, tal consulta podría ahorrar una gran cantidad de esfuerzo y de dinero, gracias al conocimiento y experiencia práctica de los consulto. res.

Estas herramientas se introducen en la oficina con objeto de ahorrar tiempo, aumentar la eficacia, obtener una mayor exactitud y dar mejor calidad en la información y en los documentos presentados, con el objetivo final de tener una mayor competitividad. Estos programas están basados en la forma tradicional de trabajar y, de hecho, niegan el principio de que la infor. mática cambiará la forma de pensar. Se supone que la utilización de estas herramientas conducirá a una ma- 
yor integración, lo que no resulta cierto en la mayor parte de los casos. Existe una gama cada vez mayor de programas baratos, poco compatibles entre sí y que no pueden ser modificados, lo cual imposibilita prácticamente la integración de los procesos y puede conducir a situaciones de gran confusión.

\section{Herramientas de CAD}

En lo referente a los sistemas CAD de asistencia en la preparación de proyectos, pueden encontrarse desarrollos más esenciales que los mencionados en el caso de programas de carácter general. La forma de resolver los problemas de proyecto en Arquitectura e Ingeniería cambiará drásticamente mediante el uso de las nuevas posibilidades del CAD e, incluso, de otras todavía desconocidas. Será en este tipo de aplicaciones donde se producirá una auténtica revolución; la posibilidad de utilizar máquinas «inteligentes", en el proceso de proyecto producirá una aproximación absolutamente distinta de la actual, siguiendo la açtual linea evolutiva en la que pueden mencionarse como escalones los sistemas de apoyo a las decisiones, la inteligencia artificial, los sistemas expertos, etc. Aunque existe ya un número importante de investigadores trabajando en estos temas, los resultados alcanzados hasta el momento deben considerarse modestos; estamos todavía en los primeros pasos del desarrollo, si bien el futuro se presenta prometedor.

La principal diferencia en la orientación de estos programas será el paso de los sistemas de procedimientos a los sistemas basados en ingeniería del conocimiento. La experiencia se introducirá en los programas de forma descriptiva, en contraposición con la forma prescriptiva utilizada actualmente, lo cual no significará la desaparición de los tipos de programa actuales, que seguirán siendo de gran eficacia en numerosas aplicaciones. Las técnicas de ingeniería del conocimiento abrirán la puerta a la utilización de parámetros "blandos", pudiendo incluir conceptos tales como sentimientos, colores, grado de satisfacción. Los programas no se basarán, exclusivamente, en algoritmos, dado que la experiencia de un usuario no puede considerarse como exclusivamente algorítmica. Los propios programas podrán tomar todo tipo de decisiones, basándose en la experiencia, para decidir entre la gama de soluciones que puedan presentarse. Todo esto conducirá a que no sea necesario una definición exacta y exhaustiva del problema.

Los principales puntos críticos son la cantidad y variedad de datos a utilizar, la velocidad de proceso y los avances en algoritmos, en lógicas de conjuntos difusos y en lenguajes de programación. Es de esperar que, en el plazo de diez años, estos sistemas nos permitan proyectar edificios en tres dimensiones, con la precisión requerida, y el poder comparar su comportamien- to físico desde distintos puntos de vista, comprobar la coherencia de los requerimientos originales, analizar las consecuencias de decisiones alternativas y permitirán, asimismo, planificar la ejecución, prever los medios materiales, humanos y económicos. Pero, por desgracia, todavía no estamos en el futuro, y los actuales arquitectos e ingenieros no pueden trabajar con herramientas que todavía no existen.

No es posible, por el momento, indicar directrices claras, pues el panorama está todavía confuso; sin embargo, sí podemos analizar la evolución posible o deseada de los equipos, de los programas e, incluso, de las organizaciones.

\section{Evolución del equipo físico y de sus prestaciones}

En cuanto al equipo físico (hardware), podría sintetizarse la situación diciendo que los equipos grandes son cada vez más baratos y los equipos baratos son cada vez más grandes. Esto permite la utilización de estaciones de trabajo independientes, que pueden constituir los eslabones de una cadena. Es posible encontrar facilidades como el uso de ventanas múltiples, sistemas de manejo de base de datos, menúes en cualquier zona de la pantalla, etc., en equipos con una amplia gama de precios.

El uso simultáneo de varias ventanas y de menúes dinámicos permiten al operador romper el ritmo secuencial de trabajo y pasar de una tarea a otra, pudiendo observar el efecto producido en las distintas ventanas. En la Fig. 1 puede observarse el aspecto de la pantalla durante una etapa intermedia del trabajo. En ella se muestran distintas vistas del objeto diseñado, pudiendo trabajar, indistintamente, sobre cualquiera de ellas, así como una serie de opciones a disposición del usuario. La utilización de iconos en lugar de mensajes puede resultar muy ilustrativo pues, en muchas ocasiones, una imagen vale más que cien palabras y, además, se evitan los problemas de idiomas.

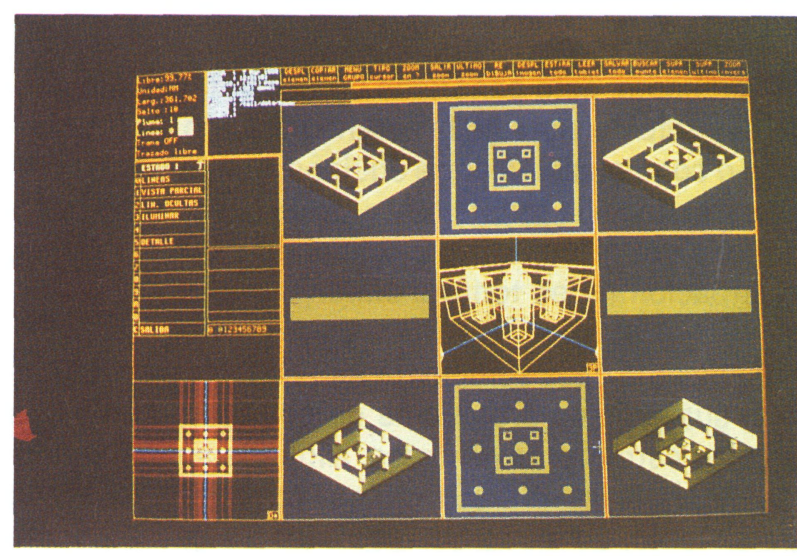

Fig. 1 
Hay una mejora constante, no sólo en las velocidades de proceso, capacidades de memoria central y periférica, métodos interactivos de comunicación, tales como tabletas gráficas, ratones, pantallas táctiles, etcétera; sino que también se están consiguiendo interesantes avances en las comunicaciones orales con el computador, más avanzadas en cuanto a sintesis de voz que en cuanto a la interpretación de órdenes habladas.

Un aspecto que puede resultar de gran interés en CAD es la utilización de procesadores en paralelo, ya que, en este tipo de aplicaciones, es frecuente tener que resolver un gran número de pequeños problemas iguales, prácticamente independientes entre sí. Otro desarrollo que puede ser importante en problemas más complejos es el de los denominados transputadores creados por la empresa inglesa INMOS.

Tienen, también, gran importancia los procesadores especialmente dedicados a aplicaciones gráficas. La incorporación de estos procesadores a pantallas gráficas de colores de gran tamaño y alta resolución han permitido crear estaciones de trabajo gráficas especializadas, muy eficientes, que pueden conectarse con sistemas potentes en el tratamiento de bases de datos. En la Fig. 2 puede contemplarse una perspectiva de un edificio íntegramente realizada por un programa de Diseño Asistido. Puede observarse el extraordinario realismo de la misma.

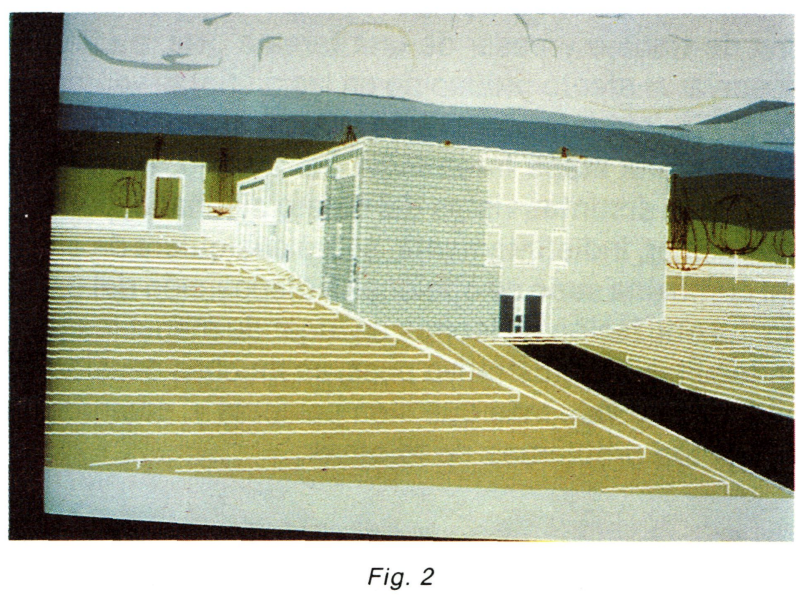

Sin embargo, este panorama no es tan favorable desde el punto de vista de la estandarización en la conexión o comunicación entre equipos, donde suelen presentarse todavía problemas y, pese al esfuerzo desarrollado por diversas organizaciones internacionales, la situación no es todavía clara.

Actualmente cada suministrador tiene sus peculiaridades, sus propios protocolos de comunicación y sus propias estructuras de datos. Algunos suministradores de sistemas "llave en mano" fomentan su propio aislamiento, de modo que sus clientes queden cautivos del sistema, dependiendo de ellos para posibles mejoras o ampliaciones y en las condiciones que el suministrador decida. Serán las grandes multinacionales quienes, probablemente, pondrán coto a estos problemas, ya que ellas mismas sufren esta falta de compatibilidad al tratar de conectar equipos de distintos fabricantes y programas de distintos orígenes. Así, por ejemplo, el sistema operativo UNIX está tratando de convertirse en un Standard de facto, si bien existen diferencias entre las versiones utilizadas en distintos sistemas.

\section{Evolución de los programas}

El problema de los programas (software) es más complejo que el de los equipos. Ya se ha hablado de la caótica situación de los programas de carácter general.

En el desarrollo de sistemas CAD se han probado, a lo largo de los años, todo tipo de principios pero, hasta el momento, sólo unos pocos de ellos han mostrado valor práctico. El punto principal es la integración. La mezcla de viejos programas con nuevas ideas resulta complicada. En teoría, sería conveniente recomenzar todo de nuevo, sin conservar más herencias del pasado que el conocimiento y la experiencia, pero esta alternativa no es realista. Hasta el momento tan sólo se han logrado programas integrados en pequeña escala, por ejemplo de ciertos tipos de construcción o para una única organización con una gama limitada de productos.

El caballo de batalla está en la información. Cualquier proceso en un entorno de proyecto o de construcción es complejo, teniendo que manejar una cantidad de información enorme. En la construcción casi todos los procesos están relacionados con casi todos los restantes, lo que significa que una única base de datos para todo el proceso es prácticamente imposible.

La información no sólo es mucha, sino también muy variada; por ejemplo, el grado de precisión que desea cada una de las partes implicadas es diferente. Cada uno de los oficios que intervienen es un mundo en sí mismo; así, la información debe estratificarse en niveles y utilizar las llamadas "ampliaciones lógicas" de la información.

Se han propuesto distintos protocolos para intercambio de datos geométricos. Por ejemplo, el sistema IGES (INITIAL GRAPHIC EXCHANGE SPECIFICATIONS) ha sido adoptado por distintos sistemas de dibujo asistido en los programas denominados de 2 y 2,5 dimensiones. Sin embargo, sus formatos son anticuados y voluminosos y hay dificultad para la transmisión de datos entre distintos sistemas. En la Fig. 3 puede verse un plano producido por un sistema de dibujo asistido por computador.

http://informesdelaconstruccion.revistas.csic.es 


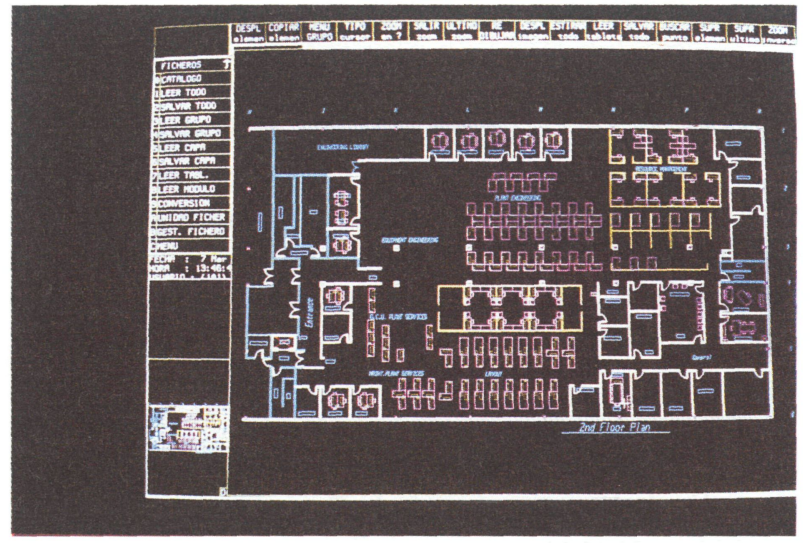

Fig. 3

No existe, todavia, ningún sistema estandarizado para el intercambio de datos geométricos entre sistemas de tres dimensiones, entre otras razones por las distintas formas, incompatibles entre sí, para representar en el espacio superficies curvas, y más aún modelos sólidos o volumétricos.
Es más probable que en el próximo futuro se produzcan estándares para tipos concretos de aplicaciones, como es el caso del sistema BDA, desarrollado para la industria de automatización alemana.

También se alega contra estos sistemas la pérdida de creatividad por parte del proyectista. En general, la mayor parte de los programas, y en particular los programas integrados son muy restrictivos; para ellos sólo pueden existir edificios con ciertas formas o formados por ciertos tipos de elementos y, cuando aparecen otros nuevos, no es posible tratarlos; incluso el hecho de que las paredes sean verticales y los suelos horizontales no puede ser considerado como un axioma.

En general, todos los sistemas pueden manejar modelos de alambres o de superficies; pero sólo los sistemas más modernos están introduciendo los modelos sólidos o de volumen. En estos últimos se describe dónde se encuentra el material en forma de partes, aristas, caras, y pueden especificarse propiedades tales como tipo de material, acabados, tolerancias, etc.

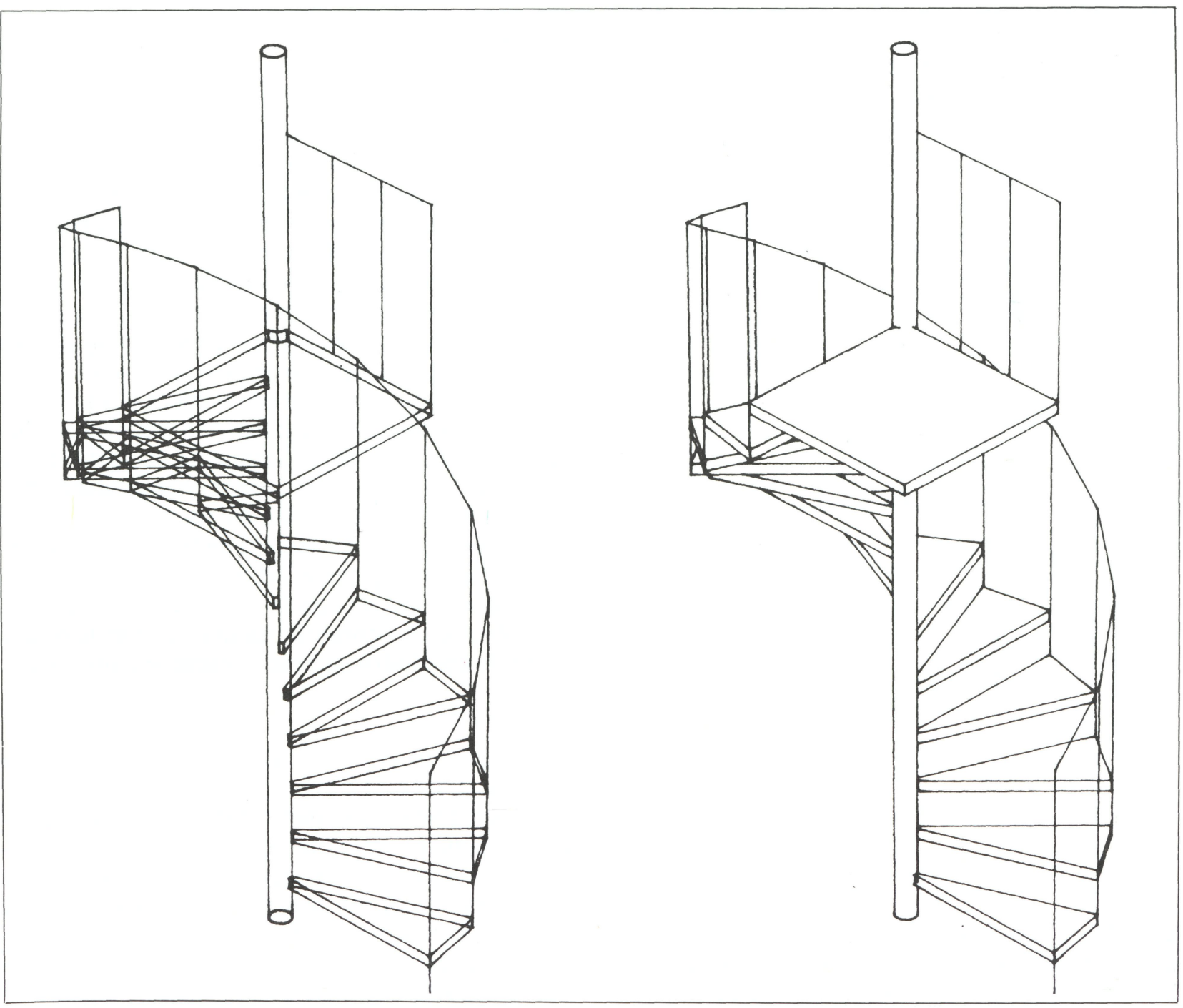


Existen distintos sistemas alternativos para definir sólidos. El más sencillo es el modelo de facetas, en el que el modelo real se aproxima mediante elementos planos. Este sistema de representación permite un tratamiento rápido de los objetos y proporciona representaciones gráficas adecuadas. En la Fig. 4 se muestran dos perspectivas de una escalera representada por el modelo de facetas. En la primera de ellas se utiliza el modelo de alambres, en tanto que en la segunda se utiliza el de superficies, eliminando las partes ocultas y dando un aspecto suave a las superficies curvas. Otro sistema más exacto es el de construcción de modelos sólidos mediante la definición y combinación de objetos sencillos descritos de un modo muy compacto. Un sistema de este tipo fue introducido en la Universidad de Okaido (Japón). Este sistema tiene el inconveniente de que cualquier reelaboración del modelo requiere volver a empezar desde el principio y, además, no permite representar, de forma paramétrica, las superficies, lo que limita grandemente sus posibilidades. El modelo que parece intuitivamente más correcto es el denominado de contornos. Este modelo permite modificaciones parciales de los objetos, por ejemplo eliminando partes de los mismos. Todos estos modelos permiten realizar un conjunto de operaciones lógicas con los objetos y determinar, de forma automática, colisiones, secciones, etc.

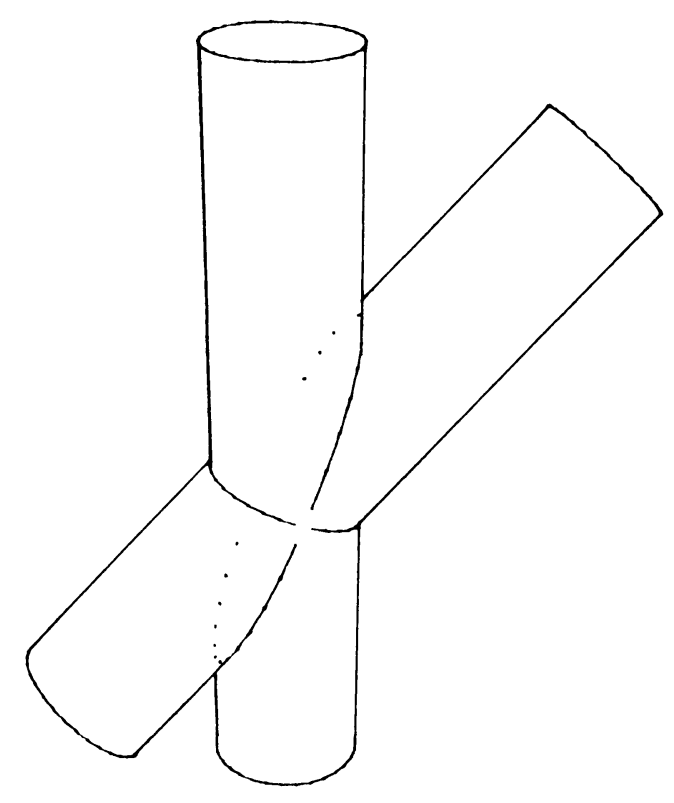

Fig. 5

En la Fig. 5 puede verse la perspectiva de la intersección de dos tubos cilindricos, cada uno de los cuales ha sido definido por separado, habiendo determinado el programa, automáticamente, su intersección.

\section{Evolución de las organizaciones}

A veces las empresas reaccionan de forma inesperada. Es frecuente la frustración de las personas que desarrollan sistemas informáticos al comprobar que sus esfuerzos en análisis y desarrolio son equivocados pues, al final, han creado un producto destinado a una organización inexistente.

La situación en Arquitectura e Ingeniería difiere, por ejemplo, de la que presenta la Banca, en la que los intereses comunes pueden definirse de un modo más simple. En nuestro ámbito, deben ser los usuarios quienes definan la clase de integración que desean y deben estudiar, con los expertos en informática, si es posible alcanzarla. Las características de la información a procesar deben ser definidas de común acuerdo. No se tratará, en general, de una base de datos común para todo el proceso, sino de un conjunto de bases de datos específicas, debiendo centrar los esfuerzos en la forma de comunicarse con y entre estas bases de datos, cada una de las cuales puede estar estructurada de forma distinta.

La industria de la construcción nunca ha sido progresista en la adopción de técnicas automáticas, si la comparamos con otras industrias como la electrónica o la mecánica; esto se debe, en parte, a que en estas últimas no existe una tradición consolidada, lo cual, desde este punto de vista, ha resultado favorable. Los procesos y la metodología de proyecto han avanzado considerablemente en los últimos años. Hoy se aplican teorias y herramientas que no existían anteriormente. En esta colaboración entre informáticos y arquitectos e ingenieros, los primeros deben ser más conscientes de las limitaciones de los programas que están desarrollando, en tanto que los últimos deben tratar de darse cuenta de la importancia de las herramientas de CAD ya existentes; así, además de preocuparse de aspectos tales como coste, precisión, rapidez en el dibujo, deben tener en cuenta la forma cómo el programa entiende las convenciones gráficas habituales, la forma de construir e, incluso, el estilo arquitectónico.

Los programas existentes en la actualidad muestran distintos grados de comprensión del proceso; así, algunos programas permiten construir un modelo plástico, en tanto que otros permiten el ensamblaje de partes tomadas de un catálogo de componentes y otros aún parecen tener alto nivel de comprensión sobre los elementos constitutivos de un edificio, del cómo pueden compaginarse, cómo ser dibujados, etc. Por supuesto, cuanto más neutral sea el modelo construido tanto mayor debe ser la creatividad del usuario. 


\section{Obtención de mediciones y presupuestos}

Uno de los aspectos de los programas del CAD que requiere también un interés especial es la obtención de mediciones y de presupuestos a partir del modelo introducido. En los sistemas actuales la parte principal radica en la obtención de planos y perspectivas, en tanto que la parte de mediciones y presupuestos tiene un carácter secundario. La complejidad de los actuales métodos para realizar las mediciones hace complicada la preparación de las salidas, por lo que los programas hoy existentes sólo producen resultados parciales.

La incorporación a las unidades de los recursos necesarios para su producción, tales como materiales, mano de obra, maquinaria, costes generales, pueden resultar de gran utilidad para realizar un estudio económico de la obra, sobre todo si se combina con la posibilidad de analizar la evolución de la obra en el tiempo o la de estudiar partes separadas del conjunto, como pueden ser suelos, departamentos, instalaciones, puertas, ventanas, etc.

Sin embargo, este tratamiento integral puede presentar algunos inconvenientes. Asi, el tratamiento de elementos poco frecuentes puede obligar a la introducción de partidas neutras especiales que pueden degradar el rendimiento del sistema. Otro posible problema puede radicar en la excesiva confianza en los resultados producidos por el computador, lo que lleva a no realizar comprobaciones sobre la misma.

\section{Conclusiones}

Los sistemas CAD aplicados en Arquitectura e Ingeniería están todavía en proceso de evolución. Se ha pasado de la etapa en la que los métodos informáticos utilizados servían para automatizar la práctica habitual a una nueva etapa, en la que el proceso completo de proyecto y construcción se abordará mediante procedimientos absolutalmente nuevos, basados en la utilización de sistemas integrados que incorporarán capacidad de decisión propia basada en la experiencia.

En los próximos años estos sistemas mejorarán considerablemente merced a la favorable evolución de las capacidades y prestaciones de los computadores, a la generalización de las estaciones gráficas especializadas, a la mejora de los sistemas de manejo de bases de datos, al desarrollo de técnicas matemáticas de modelos de representación en volumen y a lenguajes de programación que facilitarán el desarrollo de sistemas expertos.

Es importante que se produzca una evolución positiva en la compatibilidad entre distintos computadores y en la formalización de protocolos para el intercambio de datos.

Debe realizarse un esfuerzo significativo en la colaboración entre los informáticos y las restantes profesiones que intervienen en el hecho constructivo con objeto de definir, de un modo conjunto, las características que deben reunir los nuevos sistemas, la forma más cómoda y correcta de describir las construcciones en toda su complejidad, la forma de llevar a cabo el análisis de los distintos aspectos del problema, la estructuración más idónea de la enorme cantidad de información a manejar y la forma más adecuada de producir resultados gráficos y documentales.

El Instituto Eduardo Torroja ha realizado una amplia labor de investigación y de difusión de los métodos de análisis de estructuras y de los de dimensionamiento y comprobación de estructuras de hormigón teniendo en cuenta las distintas causas de no-linealidad. También ha investigado sobre métodos interactivos de introducción de datos y de dibujo de objetos tridimensionales. Actualmente ha decidido investigar en varios de los distintos problemas que plantea el desarrollo de sistemas de CAD integrados descritos anteriormente. Para ello ha planteado un proyecto de investigación así como la colaboración con distintas empresas y organismos para aplicar los resultados de la investigación en distintos sistemas comercializables.

\section{Agradecimientos}

Deseamos expresar nuestro agradecimiento a la firma ICT de Madrid por la cesión de las figuras 1 a 3 , las cuales han sido realizadas con programas de Diseño Asistido por Computador y de Dibujo Asistido por Computador, distribuidos por dicha firma.

\section{Bibliografía recomendada}

En el texto anterior no se han citado las fuentes bibliográficas consultadas; estas fuentes, así como otras obras consideradas de interés, son las que se relacionan a continuación:

1. CHASEN, Sylvan.: "Geometric Principles and Procedures for Computer Graphics Applications". Prentice Hall, Inc. New Jersey, 1978, 241 págs.

2. ENCARNAÇAO, J.; SCHLECTENDAHL, E. G.: “Computer Aided Desing", Springer-Verlag, 1983, 346 págs.

3. FOLEY, JAMES D.; VAN DAM. ANDRIES: «Fundamentals of Interactive Computer Graphics". Addisson-Werley Publishing Company, Julio 1984, 664 págs. 
4. GILOI, W. K.: «Interactive Computer Graphics». Prentice Hall, Fnc. New Jersey, 1978, 393 págs.

5. NEWMAN, W. M.; SPROULL, R.: "Principles of Interactive Computer Graphics». McGraw Hill, 1981, 541 págs.

6. SANTO, H.: «Métodos Gráficos e Geometría Computacionais». Vol. 1, Lisboa 1985, 299 págs.

7. "Knowledge Engineering and Computer Modelling in CAD». Proceedings of CAD 86. Ed. Alison Smith, London, s-5 Sep. 1986, 476 págs.

8. NILSSON, N. J.: "Principios de Inteligencia Artificial». Ed. Díaz de Santos, 1987, 422 págs.
9. WINSTON, P. H.: «Artificial Intelligence». Addison-Wesley Publishing Company, 1984, 524 págs.

10. SIMONS, G. L.: "Introducción a la Inteligencia Artificial». Ed. Diaz de Santos, S. A., 1987, 257 págs.

11. CHADWICK, M. y HANNAH, J. A.: "Sistemas Expertos para Ordenadores Personales». Sigma Press, 1986, 248 páginas.

12. RECUERO, A.; GUTIERREZ, J. P. y RIO, O.: “Dibujo de Perspectivas, asistido por computador, utilizando dispositivos trazadores gráficos". Monografía del IETCC número 391, Madrid, 1987.

\section{JOSE MARIA AGUIRRE GONZALO}

Con profundo pesar notificamos a nuestros lectores el fallecimiento de don José M. ${ }^{\text {a }}$ Aguirre, Presidente durante años del Consejo Técnico Administrativo del Instituto Eduardo Torroja de la Construcción y del Cemento, ocurrido el 7 de abril del presente año.

Ha sido tan evidente su desbordante personalidad en los aspectos tanto financiero, como político, técnico y social, que nos eximen de profundizar en su talla universal en estos campos.

Para los lectores de esta Revista conviene recordar que, en 1934, Eduardo Torroja, José M. ${ }^{a}$ Aguirre y Modesto López Otero fundaron el Instituto tratando, con gran ilusión, de mejorar las técnicas de la construcción.

En noviembre de 1974 organizó y presidió la 2. ${ }^{a}$ Asamblea General del Centro, coincidiendo con el 40 Aniversario de la fundación del mismo.

En todo momento demostró gran interés por el futuro del Instituto, dando impulso a su desarrollo. 